\title{
Liberação de Benzoato de Cálcio de Filmes de Alginato de Sódio Reticulados com Íons Cálcio
}

\author{
Franciele R. B. Turbiani, Theo G. Kieckbusch \\ Departamento de Termofluidodinâmica, UNICAMP \\ Marcelino L. Gimenes \\ Departamento de Engenharia Química, UEM
}

\begin{abstract}
Resumo: Biofilmes confeccionados à base de alginato de sódio foram reticulados com íons $\mathrm{Ca}^{++}$provenientes de duas fontes, cloreto e benzoato de cálcio, e continham glicerol como plastificante. Inicialmente, devido ao alto poder gelificante do $\mathrm{Ca}^{++}$, um filme de baixo grau de reticulação foi confeccionado por casting ( $1^{\circ}$ estágio). Esse filme sofreu uma reticulação complementar por imersão em uma solução contendo de 3 a $7 \%$ de $\mathrm{CaCl}_{2} \cdot 2 \mathrm{H}_{2} \mathrm{O}$, além de glicerol ( $2^{\circ}$ estágio). A liberação de benzoato de cálcio foi avaliada em diferentes concentrações de agente ativo no filme e dois níveis de reticulação do alginato. O mecanismo envolvido no processo de difusão foi investigado usando o modelo da Lei de Potência. Os resultados indicaram que a difusão de benzoato de cálcio em filmes de alginato apresenta características de comportamentos Fickiano e não-Fickiano. Os coeficientes de difusão efetivos obtidos usando a solução em série derivada da $2^{\mathrm{a}}$ Lei de Fick são próximos aos valores obtidos pela solução em tempos curtos, com valores de difusividade efetiva do benzoato variando de 3 a $5 \cdot 10^{-7} \mathrm{~cm}^{2} / \mathrm{s}$. Os valores de difusividade diminuíram com o aumento da intensidade de reticulação e aumentaram com a concentração de benzoato no filme.
\end{abstract}

Palavras-chave: Alginato, biofilmes, benzoato de cálcio, coeficiente de difusão, reticulação, liberação.

\section{Release of Calcium Benzoate from Films of Sodium Alginate Crosslinked with Calcium Ions}

Abstract: Alginate-based biofilms were reticulated with $\mathrm{Ca}^{++}$supplied by two sources, calcium chloride and benzoate, and using glycerol as plasticizer. The strong gelling power of the $\mathrm{Ca}^{++}$ions hindered smooth casting procedures, so that films with low degree of reticulation were initially manufactured ( $1^{\text {st }}$ stage). These films were further crosslinked with an excess of $\mathrm{Ca}^{++}$by immersion in a solution of 3 to $7 \%$ of $\mathrm{CaCl}_{2} \cdot 2 \mathrm{H}_{2} \mathrm{O}\left(2^{\text {nd }}\right.$ stage $)$. The release of sorbate was evaluated considering different active agent concentrations in the film and two levels of alginate crosslinking. The mechanism involved in the diffusional process was investigated using the Power Law Model. The results indicated that potassium sorbate diffusion in alginate films has characteristics of Fickian and non-Fickian behavior. Effective diffusion coefficients obtained using the solution in series derived from Fick's Second Law are close to values obtained with the short-time solution, with effective diffusivities varying from 3 to $5 \times 10^{-7} \mathrm{~cm}^{2} / \mathrm{s}$. The diffusivity values decreased with the degree of reticulation and increase with benzoate concentration in the film.

Keywords: Alginate, biofilms, calcium benzoate, diffusion coefficient, crosslinking, release.

\section{Introdução}

Os alimentos, de forma geral, são altamente perecíveis. Deste modo, inúmeros são os problemas relacionados à conservação dos mesmos, seja na forma in natura ou como produtos processados. Geralmente uma combinação de métodos são utilizados, sendo indispensável a proteção física do alimento, na forma de embalagens adequadas $^{[1]}$.

A produção e aplicação de biofilmes com atividade antimicrobiana podem ser classificadas como uma tecnologia de liberação controlada. Este procedimento é utilizado para liberar substâncias ativas, como medicamentos, pesticidas, conservantes e fragrâncias em doses controladas, melhorando a eficácia, segurança e conveniência de sua utilização.

Nos últimos anos, sistemas de liberação controlada tornaram parte de uma ampla categoria de novos conceitos de embalagem de alimentos conhecidos como "materiais de embalagem ativa". Promissores sistemas de embalagens ativas são baseadas na incorporação de substâncias antimicrobianas na estrutura da embalagem de alimentos, a fim de controlar o crescimento de microorganismos indesejável na superfície dos alimentícios ${ }^{[2-6]}$.
Uma vez que a eficiência antimicrobiana dos filmes é baseada na migração de substâncias ativas, o conhecimento da difusividade desses compostos no interior do filme é um fator determinante no desenvolvimento de sistemas antimicrobianos de embalagem de alimentos. A difusividade pode ser afetada pelo tamanho e forma das moléculas de substância ativa, a estrutura do polímero (tamanho dos poros, flexibilidade da cadeia polimérica e densidade) e condições ambientais ( $\mathrm{pH}$, atividade de água e temperatura). Os parâmetros de composição do filme, tais como, concentração de polímero, tipo e concentração de plastificante e a presença de componentes como lipídios e agentes ativos podem influenciar o processo de difusão.

Alginatos são de grande interesse como potencial componente estrutural de filmes e coberturas por causa de suas propriedades coloidais únicas ${ }^{[7,8]}$. É um carboidrato coloidal hidrofílico extraído com álcali diluído de várias espécies de algas marrons. É o único polissacarídeo que contém, naturalmente, grupos carboxílicos em cada constituinte residual, e possui várias habilidades funcionais ${ }^{[9]}$. A propriedade mais usada e única em alginatos é sua habilidade de reagir com cátions polivalentes, especialmente íons cálcio, para produzir géis fortes ou polímeros insolúveis ${ }^{[10,11]}$. 
Dependendo do grau de reticulação, o intumescimento da estrutura tridimensional do alginato na presença de solventes é significativamente reduzido, resultando em uma diminuição na permeabilidade para diferentes solutos. Como consequência, a liberação do agente incorporado é lenta, permitindo que estes sistemas sejam utilizados na liberação controlada de substâncias.

A produção de filmes de alginato de cálcio é uma operação complexa e depende do tipo de alginato utilizado, do grau de conversão entre o alginato e o cálcio, da fonte de íons de cálcio (cloreto, fosfato, lactato, etc) e do método de preparação ${ }^{[12]}$. O principal objetivo deste estudo foi avaliar a liberação de benzoato a partir de filmes de alginato, influenciados pela concentração do agente ativo e pelo grau de reticulação do filme.

\section{Materiais e Métodos}

\section{Materiais}

Para a confecção dos filmes, foi utilizado alginato de sódio de média viscosidade extraído de alga Macroscystis Pyrifera (Synth P.A., Brasil). Cloreto de cálcio dihidratado (Merck ACS, USA) foi utilizado como agente reticulante, glicerol (Synth P.A., Brasil) como agente plastificante e benzoato de cálcio trihidratado (Avocado, USA) como agente ativo e auxiliar na reticulação.

\section{Preparação dos filmes de alginato}

Os filmes de alginato foram obtidos segundo a técnica de casting que consiste na preparação da solução filmogênica e aplicação da mesma em um suporte. Inicialmente, foi produzido um filme de baixo grau de reticulação com $\mathrm{Ca}^{++}$, cuja solução filme-formadora continha alginato, plastificante e cloreto e/ou benzoato de cálcio. Essa etapa inicial foi denominada de $1^{\circ}$ estágio. Primeiramente, solubilizou-se o glicerol (0,6 g glicerol/g alginato) em $400 \mathrm{~mL}$ de água destilada a $70{ }^{\circ} \mathrm{C}$ e, em seguida, adicionou-se o alginato de sódio $(1,5 \%)$. $\mathrm{Na}$ sequência, adicionou-se lentamente $30 \mathrm{~mL}$ da solução préreticuladora contendo $\mathrm{Ca}^{++}$, mantendo a temperatura em $70{ }^{\circ} \mathrm{C}$, sob intensa agitação. Alíquotas de $70 \mathrm{~g}$ dessa solução foram transferidas para placas de polipropileno (diâmetro $=14 \mathrm{~cm}$ ) e levadas a uma estufa com circulação de ar, a $40{ }^{\circ} \mathrm{C}$, por 16 horas, para uma gelificação incipiente e formação de um filme por evaporação.

Esse filme foi submetido a uma reticulação complementar por imersão em um banho a temperatura ambiente contendo $50 \mathrm{~mL}$ de uma solução de $\mathrm{CaCl}_{2} 2 \mathrm{H}_{2} \mathrm{O}$ e/ou benzoato de cálcio, além do plastificante $(5 \% \mathrm{v} / \mathrm{v})$. Pesquisas preliminares com tempos de imersão variando de 5 a 60 minutos indicaram que 30 minutos de contato produz filmes de maior resistência mecânica e de menor solubilidade em água, e esse tempo foi adotado em todas as formulações.

Após a reticulação do $2^{\circ}$ estágio, os filmes foram retirados do banho e mantidos sobre uma base plana por três horas a temperatura ambiente para uma uniformização de sua concentração interna. Para o acabamento final, esses filmes foram submetidos à secagem em estufa ( $30^{\circ} \mathrm{C} / 20$ horas). Uma descrição mais detalhada deste processo pode ser encontrada em trabalho previamente publicado ${ }^{[13]}$.

Antes de sua caracterização, os filmes foram armazenados por 72 horas em dessecadores a uma umidade relativa de 52\% (solução saturada de nitrato de magnésio) e temperatura de $25 \pm 1{ }^{\circ} \mathrm{C}$ para uniformizar seu conteúdo de umidade.

Apenas filmes com boa aparência, avaliados subjetivamente levando em consideração parâmetros como homogeneidade (ausência de partículas insolúveis e coloração uniforme), continuidade (ausência de rupturas ou fissuras após a secagem), flexibilidade, facilidade de desprendimento do suporte e facilidade de manuseio foram submetidos à caracterização física.

\section{Caracterização física dos filmes}

\section{Espessura}

A espessura, $\delta$, foi determinada utilizando um micrômetro digital (MITUTOYO, modelo MDC-25S, Japão, com resolução de $0,001 \mathrm{~mm})$, realizando no mínimo 10 medidas em cada filme.

\section{Conteúdo de umidade}

A umidade foi determinada em triplicata para cada formulação de filme, de acordo com Rhim et al. ${ }^{[14]}$. A massa inicial de discos cortados dos filmes, de $2,5 \mathrm{~cm}$ de diâmetro foi quantificada e as amostras submetidas à secagem a $105^{\circ} \mathrm{C}$ por 24 horas em estufa a vácuo (Lab-Line, Squaroid, EUA) e a massa seca quantificada novamente. O conteúdo de umidade foi expresso como fração da massa inicial do filme, $\omega$, ou em \% da massa (base úmida).

\section{Massa solubilizada em água}

A massa solubilizada em água, $M S$, foi determinada em triplicata, de acordo com Gontard et al. ${ }^{[15]}$. A massa total de amostras do filme foi quantificada $\left(m_{\mathrm{o}}\right)$ e o material imerso em excesso de água destilada sob agitação branda a $25 \pm 1^{\circ} \mathrm{C}$ por 24 horas, utilizando-se um Shaker Bath Orbit (Lab-Line, EUA). A massa seca final $\left(m_{f}\right)$ foi determinada submetendo-se esta amostra à secagem $\left(105^{\circ} \mathrm{C} / 24\right.$ horas $)$. A massa solubilizada foi expressa em função da massa seca inicial, conforme Equação 1:

$$
M S=\frac{m_{o}(1-\omega)-m_{f}}{m_{o}(1-\omega)} .100
$$

\section{Grau de intumescimento (swelling)}

A massa total inicial de uma amostra cortada do filme, de $2,5 \times 2,5 \mathrm{~cm}$, foi quantificada $\left(m_{\mathrm{o}}\right)$ e o material imerso em água destilada sob leve agitação, a $25{ }^{\circ} \mathrm{C}$. Em determinados períodos de tempo, a amostra foi retirada da água, o excesso de umidade superficial das amostras foi removido, colocando o filme entre duas folhas de papel de filtro, sua massa total $\left(m_{u}\right)$ determinada e a amostra retornava à água. $\mathrm{O}$ grau de intumescimento $(\mathrm{GI})$ foi calculado pela relação $\left(m_{u}-m_{\mathrm{o}}\right) /\left(m_{\mathrm{o}}\right)^{[16]}$. Essas determinações foram realizadas em triplicata para cada formulação de filme.

\section{Propriedades mecânicas}

As propriedades mecânicas dos filmes foram obtidas segundo o método D882 ${ }^{[17]}$, utilizando um texturômetro TA.XT2 (Stable Microsystems, Inglaterra), em ambiente dotado de ar condicionado à temperatura de $25 \pm 1{ }^{\circ} \mathrm{C}$ e umidade relativa de $55 \pm 3 \%$.

Amostras de dimensões de 25,4 por $100 \mathrm{~mm}$ foram ajustadas às garras do equipamento. A separação inicial foi de $50 \mathrm{~mm}$ e a velocidade de tração foi de $0,8 \mathrm{~mm} / \mathrm{s}$. A tensão na ruptura (força máxima no momento da ruptura/área de seção transversal inicial do filme) e o alongamento na ruptura (porcentagem em relação ao comprimento inicial) foram determinados diretamente usando o software Texture Expert V.1.15 da Stable Microsystems.

\section{Permeabilidade ao vapor de água}

A permeabilidade ao vapor de água $(P V A)$ foi determinada gravimetricamente, de acordo com o método E96-95 ${ }^{[18]}$, utilizando pequenas células de alumínio a $25 \pm 1{ }^{\circ} \mathrm{C}$. Amostras circulares de filmes, $8 \mathrm{~cm}$ diâmetro, foram hermeticamente fixadas na borda da célula com parafina, e a célula permaneceu no interior de um dessecador com umidade relativa constante de $75 \%$, obtida com uma solução saturada de cloreto de sódio. A área da superfície livre exposta dos filmes para permeação de vapor foi $44 \mathrm{~cm}^{2}$. Cloreto de cálcio granulado preencheu o fundo da célula, mantendo um micro-ambiente a $0 \%$ de UR. As células foram pesadas diariamente com uma balança 
analítica durante sete dias. O valor da PVA foi calculado através da Equação 2. Todas as determinações foram feitas em triplicata.

$$
P V A=\frac{\stackrel{G}{G} \cdot \delta}{A_{e} \cdot \Delta P_{\mathrm{H}_{2} \mathrm{O}}}
$$

onde: $P V A$ é a permeabilidade ao vapor de água $\left[(\mathrm{g} . \mathrm{mm}) /\left(\mathrm{m}^{2} . \operatorname{dia} . \mathrm{kPa}\right)\right]$; $\delta$ é a espessura inicial do filme $[\mathrm{mm}] ; \stackrel{o}{G}$ é a taxa constante de ganho de massa [g/dia]; $\mathrm{A}_{\mathrm{e}}$ é a área de permeação do filme; $\Delta P_{\mathrm{H} 2 \mathrm{O}}$ é a diferença de pressão parcial do vapor de água no ambiente próximo às duas faces do filme na temperatura do ensaio $[\mathrm{kPa}]$.

\section{Cinética da liberação do benzoato}

Os ensaios de liberação foram realizados utilizando-se amostras de filmes ativos de dimensões $4 \times 4 \mathrm{~cm}$ (massa media igual a $0,15 \mathrm{~g}$ ). Como sorvedouro para a liberação, foram utilizados volumes de $25 \mathrm{~mL}$ de água destilada a temperatura de $25 \pm 0,5^{\circ} \mathrm{C}$. Os béqueres contendo a água foram dispostos em um shaker e mantidos sob agitação durante o ensaio, a fim de diminuir uma possível resistência por convecção de massa. No tempo zero, a amostra de filme era mergulhada no primeiro copo e, após um intervalo de tempo prédeterminado, era rapidamente transferida para o segundo copo, e assim por diante, de maneira que a massa acumulada de benzoato na solução de cada béquer corresponde a um ponto da curva de liberação. Com a utilização de quantidades renovadas de água, garante-se que a concentração de benzoato nas interfaces do filme seja zero, além de se obter amostras bem definidas de soluto liberado nos intervalos de tempo. Para a determinação da concentração de benzoato de cálcio em solução aquosa, foi utilizado um espectrofotômetro (HP, modelo 8453, EUA) equipado com controlador de temperatura HP $89090^{\mathrm{A}} \mathrm{e}$ software UV-Visible ChemStation. A concentração de benzoato era lida em curva de calibração.

O primeiro minuto desse ensaio é crítico, pois testes preliminares indicaram a necessidade de fazer a transposição do filme nos copos a cada 5 segundos (até 35 segundos) e então a cada 10 segundos. Nesse período, a amostra de filme era mantida presa a uma pinça. Após essa etapa inicial, os intervalos eram maiores sendo mais fácil controlar a retirada e transferência do filme para o copo seguinte.

A massa acumulada de benzoato de cálcio liberada no tempo $t\left(M_{t}\right)$ e os valores de fração de benzoato liberado $\left(M_{t} / M_{\infty}\right)$ foram calculados e locados em gráfico em função do tempo. A quantidade de benzoato que poderia ser liberada em um tempo infinito $\left(M_{\infty}\right)$ foi considerada como a massa total dessorvida a partir da qual não se constatou variação de concentração. A espessura média do filme foi determinada antes e no final de cada ensaio.

\section{Parâmetros da Difusão}

A $2^{a}$ Lei de Fick da difusão foi usada para modelar a liberação de benzato de cálcio de filmes de alginato. De acordo com as condições experimentais e assumindo a espessura do filme constante, $\delta$, a seguinte equação para uma placa plana pode ser adaptada de $\mathrm{Crank}^{[19]}$ para a fração de benzoato de cálcio liberada em função do tempo:

$$
\frac{M_{t}}{M_{\infty}}=1-\frac{8}{\pi^{2}} \sum_{n=0}^{\infty} \frac{1}{(2 n+1)^{2}} \exp \left(-(2 n+1)^{2} \frac{\pi^{2} D_{e f}}{2 \delta^{2}} t\right)
$$

onde $\mathrm{D}_{\text {ef }}$ é a difusividade efetiva média do benzoato de cálcio, assumida constante.

Uma solução simplificada da $2^{\mathrm{a}}$ Lei de Fick, a solução para tempos curtos, pode ser utilizada para o ajuste dos pontos experimentais iniciais, quando menos de $60 \%$ da massa de soluto é liberada ${ }^{[19]}$ :

$$
\frac{M_{t}}{M_{\infty}}=\sqrt{\frac{16 D_{e f} t}{\pi \delta^{2}}}
$$

Matrizes poliméricas, no entanto, estão propensas a sofrer relaxamento na estrutura sob a influência solventes penetrantes ou moléculas de soluto e mecanismos de transporte anômalo prevalece para as situações em que $\mathrm{M}_{\mathrm{t}} / \mathrm{M}_{\infty}<0.6^{[20]}$. Esta migração não-Fickiana de solutos pode ser modelada pela conhecida Lei da Potência ${ }^{[19]}$ :

$$
\frac{M_{t}}{M_{\infty}}=k t^{n}
$$

onde k é uma constante que caracteriza o sistema macromolecular e n é o expoente de difusão característico do mecanismo de liberação. Valores de $n$ normalmente se situam entre 0,5 e 1,0. O limite de $n=0,5$ corresponde ao processo de difusão Fickiana e as Equações 3 ou 4 devem ser usadas.

\section{Planejamento dos ensaios}

A pesquisa considerou diferentes concentrações relativas de benzoato e cloreto de cálcio, tanto no $1^{\circ}$ como no $2^{\circ}$ estágio ${ }^{[13]}$. O presente trabalho contempla apenas as características físico-químicas de 10 formulações de filmes que apresentaram boa aparência e funcionalidade, e dentre estas, foram selecionados 4 filmes que continham quantidades apreciáveis de benzoato que foram submetidos a ensaios de liberação. A denominação destes filmes em relação à fonte e a quantidade de cálcio incorporada no $1^{\circ}$ estágio (F1 a F\%) está apresentada na Tabela 1. Cada uma destas formulações básicas foi submetida a duas intensidades de reticulação no $2^{\circ}$ estágio por imersão em soluções de $3 \%$ ou de $7 \%$ de $\mathrm{CaCl}_{2} .2 \mathrm{H}_{2} \mathrm{O}$, recebendo então o índice 3 ou 7, respectivamente.

\section{Análise estatística dos resultados}

A avaliação estatística dos resultados obtidos foi realizada, por meio do programa STATISTICS, versão 5 - 97, empregando-se Teste de Tukey para comparação das médias das propriedades a um nível de $95 \%$ de confiança.

\section{Resultados e Discussão}

Filmes de alginato produzidos em dois estágios de reticulação eram homogêneos, transparentes e visualmente atrativos. $\mathrm{O}$ uso de uma quantidade limite de agente reticulante $\left(1^{\circ}\right.$ estágio) antes da consolidação total da estrutura do filme foi necessário para se conseguir uma adequada manuseabilidade dos filmes. Estes filmes de baixo grau de reticulação eram muitos flexíveis e completamente solúveis em água. Zactiti e Kieckbusch ${ }^{[21]}$ também obtiveram filmes de alginato totalmente solúveis em água, quando manufaturados sem o estágio de reticulação final. Filmes de alginato completamente solúveis também foram obtidos por Pavlath et al. ${ }^{[22]}$ pela adição de $0,18 \mathrm{~g} \mathrm{CaCl}_{2} \cdot 2 \mathrm{H}_{2} \mathrm{O} / \mathrm{g}$ alginato em um único estágio de procedimento. Formulações semelhantes as do presente trabalho contendo alginato de sódio foram apresentadas por Rajsharad e Kamble ${ }^{[23]}$ para aplicação como filmes,

\begin{tabular}{|c|c|c|c|}
\hline \multirow{2}{*}{\multicolumn{2}{|c|}{ Filme $\frac{\mathrm{mg} \mathrm{Ca}^{++} / \mathrm{g} \text { alginato }}{\text { (fonte: cloreto) }}$}} & \multirow{2}{*}{$\frac{\mathrm{mg} \mathrm{Ca}^{++} / \mathrm{g} \text { alginato }}{\text { (fonte: benzoato) }}$} & \multirow{2}{*}{$\frac{\mathrm{Ca}^{++} \text {total }}{\mathrm{mg} \mathrm{Ca}^{++} / \mathrm{g} \text { alginato }}$} \\
\hline & & & \\
\hline F1 & - & 0,70 & 0,70 \\
\hline F2 & - & 0,78 & 0,78 \\
\hline F3 & - & 0,83 & 0,83 \\
\hline F4 & 0,36 & 0,24 & 0,60 \\
\hline F5 & 0,36 & 0,30 & 0,66 \\
\hline
\end{tabular}
coberturas, liberação controlada de fármacos, dentre outros.

Tabela 1. Concentração de $\mathrm{Ca}^{++}$em filmes de alginato ( $1^{\circ}$ Estágio) confeccionados com cloreto e/ou benzoato de cálcio. 
Para a definição da formulação mais adequada dos filmes pesquisados, levou-se em consideração a quantidade de $\mathrm{Ca}^{++}$ adicionada, valores intermediários de solubilidade dos filmes em água (em torno de 17\%) e conteúdo não nulo de benzoato de cálcio nas formulações. Uma descrição mais detalhada deste processo por ser encontrada em trabalho previamente publicado ${ }^{[13]}$.

A definição da quantidade de cálcio a ser utilizada na otimização da concentração da solução filmogênica teve como objetivo principal a incorporação da máxima quantidade de benzoato de cálcio aceita pela estrutura do filme. O cloreto de cálcio mostrou ser um agente reticulante muito potente e em concentrações superiores a $0,5 \mathrm{mg}$ $\mathrm{Ca}^{++} / \mathrm{g}$ alginato os filmes apresentavam heterogeneidades, decorrentes de gelificação local durante a mistura dos componentes. Quando o benzoato era usado como fonte única de cálcio no $1^{\circ}$ estágio, essa concentração limite, em termos de $\mathrm{Ca}^{++}$podia ser ampliada, sem interferir na aparência do filme. O valor máximo encontrado para a adição do antimicótico à solução base foi o equivalente a $0,83 \mathrm{mg}$ $\mathrm{Ca}^{++} / \mathrm{g}$ alginato, pois conteúdos superiores tendiam a incorporar bolhas durante a intensa agitação necessária no $1^{\circ}$ estágio (em torno de 2000 rpm). Por outro lado, ensaios feitos durante a confecção de filmes contendo $\mathrm{Ca}^{++}$adicionado na forma de benzoato e cloreto mostraram que, soluções filmogênicas contendo até um total de $0,66 \mathrm{mg} \mathrm{Ca}^{++} / \mathrm{g}$ alginato produziam filmes de boa aparência e nenhuma gelificação incipiente durante o preparo no $1^{\circ}$ estágio.

Não foi possível a adição de benzoato de cálcio no $2^{\circ}$ estágio de reticulação devido a sua baixa solubilidade, que induzia a recristalização do mesmo sobre o filme. Alem disso composições contendo benzoato de cálcio apenas no $1^{\circ}$ estágio produziam filmes mais compactos e de melhor aspecto. Desta maneira, os Filmes F1 e $\mathrm{F} 3$, reticulados no segundo estágio com soluções contendo 3 e com $7 \%$ de $\mathrm{CaCl}_{2} \cdot \mathrm{H}_{2} \mathrm{O}$ foram selecionados para serem submetidos a ensaios de liberação de benzoato.

\section{Caracterização física dos filmes}

Filmes reticulados com $\mathrm{Ca}^{2+}$ apresentaram uma espessura média de $0,087 \pm 0,005 \mathrm{~mm}$ e excelente funcionalidade. Os filmes foram facilmente removidos do suporte, apresentaram boa flexibilidade, eram transparentes e lisos. As características físicas dos filmes obtidos com benzoato de cálcio são apresentadas na Tabela 2. Um aumento na concentração de $\mathrm{Ca}^{2+}$ no $2^{\circ}$ estágio resulta em um aumento no grau de reticulação ${ }^{[13]}$, e, como consequência, a solubilidade em água diminuiu, a resistência à tração aumentou e o alongamento diminui.
Uma baixa solubilidade de filmes biodegradáveis é um dos requisitos mais importantes para aplicação em alimentos e fármacos. No entanto, a maioria dos estudos publicados com biopolímeros aborda essencialmente as características mecânicas e propriedades de barreira dos filmes ${ }^{[24,25]}$.

Observa-se um aumento da fração de material solúvel com o aumento no teor de cloreto na solução de tratamento do $2^{\circ}$ estágio. Esse aumento consistente pode ser justificado pela possível formação de uma reticulação compacta na superfície dos mesmos, devido ao contato com excesso de $\mathrm{Ca}^{2+}$, conforme já verificado por Al-Musa et al. ${ }^{[26]}$. Essa camada dificulta a difusão de mais cálcio para o interior da matriz filmogênica.

Os valores de grau de intumescimento obtidos para os filmes são muito baixos se comparados a dados de outros biopolímeros. $\mathrm{O}$ efeito do aumento da concentração de $\mathrm{Ca}^{++}$na solução reticuladora sobre a diminuição do GI é considerável. Zactiti e Kieckbusch ${ }^{[27]}$ usando uma formulação com $3 \% \mathrm{CaCl}_{2} .2 \mathrm{H}_{2} \mathrm{O}$ e $0,7 \%$ de glicerol, que era pulverizado sobre as faces do filme, no $2^{\circ}$ estágio, obtiveram GI da ordem de 0,70 . A comparação entre esses valores indica que o processo por imersão além de ser mais simples, é mais eficiente em termos de controle do intumescimento.

O aumento da concentração de cálcio na formulação melhorou significativamente a tensão de ruptura do filme, reflexo de uma reticulação mais eficiente, provavelmente na região da superfície. Esse aumento da tensão na ruptura após o tratamento com cálcio pode ser explicado pela consolidação da reticulação entre os grupos carboxílicos presentes na molécula de alginato e os íons $\mathrm{Ca}^{++[22]}$. Rhim $^{[25]}$ também observou um aumento na tensão na ruptura depois do tratamento com $\mathrm{CaCl}_{2}$. Os valores de tensão de ruptura encontrados são ligeiramente inferiores aos reportados por Zactiti e Kieckbusch ${ }^{[21]}$, que usaram, entretanto, apenas $0,7 \%$ de glicerol na solução do $2^{\circ}$ estágio, isto é, uma quantidade muito menor de plastificante.

Os valores de alongamento são baixos comparados aos de outros biofilmes, em todas as condições avaliadas. Em nível molecular, a presença do íon $\mathrm{Ca}^{2+}$ é considerada como um obstáculo à rotação (movimento) das cadeias de alginato, diminuindo a mobilidade das mesmas e consequentemente a capacidade do filme em alongar-se ${ }^{[28]}$. Resmuñan-López e Bodmeier ${ }^{[29]}$ verificaram que a porcentagem de alongamento diminui após reticulação dos filmes de alginato com cálcio, tendo utilizado concentrações entre 1 e $15 \% \mathrm{Ca}^{2+}$. Além disso, eles constataram que a partir de $20 \%$ de cálcio na solução, os filmes apresentaram maior alongamento quando comparados aos filmes reticulados com menores concentrações de cálcio. Embora os autores

Tabela 2. Efeito da concentração cálcio na solução de tratamento do $2^{\circ}$ Estágio, nas propriedades físicas de filmes de alginato.

\begin{tabular}{|c|c|c|c|c|c|}
\hline Filme & $\begin{array}{l}\text { Umidade } \\
\left(\mathrm{g} / 100 \mathrm{~g}_{\text {filme }}\right)\end{array}$ & $\begin{array}{c}\text { Solubilidade } \\
\qquad \%)\end{array}$ & GI & $\begin{array}{c}\text { Tensão na ruptura } \\
\text { (MPa) }\end{array}$ & $\begin{array}{c}\text { Alongamento } \\
(\%)\end{array}$ \\
\hline $\mathrm{F}_{3}$ & $13,7 \pm 0,33^{\mathrm{a}}$ & $19,5 \pm 0,55^{\mathrm{a}, \mathrm{b}}$ & $0,50 \pm 0,027^{b}$ & $71,2 \pm 2,0^{\mathrm{a}}$ & $0,71 \pm 0,12^{\mathrm{a}}$ \\
\hline $\mathrm{F} 1_{7}$ & $16,5 \pm 0,73^{b, c, d}$ & $28,1 \pm 0,17^{\mathrm{d}}$ & $0,38 \pm 0,039^{a}$ & $86,2 \pm 5,1^{\mathrm{a}, \mathrm{b}}$ & $1,57 \pm 0,33^{\mathrm{a}, \mathrm{b}}$ \\
\hline $\mathrm{F}_{2}$ & $13,7 \pm 0,31^{a}$ & $19,5 \pm 0,88^{a, b}$ & $0,52 \pm 0,037^{b}$ & $95,2 \pm 3,8^{b}$ & $2,09 \pm 0,27^{\mathrm{b}, \mathrm{c}, \mathrm{d}}$ \\
\hline $\mathrm{F} 2{ }_{7}$ & $17,2 \pm 0,36^{\mathrm{d}}$ & $22,7 \pm 1,04^{b, c}$ & $0,37 \pm 0,021^{a}$ & $110,0 \pm 9,9^{\mathrm{c}}$ & $3,10 \pm 0,27^{d}$ \\
\hline $\mathrm{F}_{3}$ & $15,6 \pm 0,83^{\mathrm{b}, \mathrm{c}}$ & $16,9 \pm 2,62^{\mathrm{a}}$ & $0,51 \pm 0,023^{b}$ & $102,5 \pm 6,3^{\mathrm{b}, \mathrm{c}}$ & $2,03 \pm 0,33^{\mathrm{b}, \mathrm{c}, \mathrm{d}}$ \\
\hline $\mathrm{F}_{7}$ & $17,3 \pm 0,15^{\mathrm{d}}$ & $21,4 \pm 0,37^{a, b, c}$ & $0,39 \pm 0,017^{\mathrm{a}}$ & $113,6 \pm 4,2^{c}$ & $3,12 \pm 0,25^{\mathrm{d}}$ \\
\hline $\mathrm{F} 4_{3}$ & $16,8 \pm 0,36^{\mathrm{c}, \mathrm{d}}$ & $18,4 \pm 1,58^{a, b}$ & $0,49 \pm 0,042^{b}$ & $95,1 \pm 2,3^{b}$ & $1,58 \pm 0,32^{\mathrm{a}, \mathrm{c}}$ \\
\hline $\mathrm{F} 4_{7}$ & $17,2 \pm 0,27^{\mathrm{d}}$ & $24,4 \pm 3,82^{\mathrm{c}, \mathrm{d}}$ & $0,37 \pm 0,029^{a}$ & $109,2 \pm 8,9^{c}$ & $3,30 \pm 0,45^{\mathrm{d}}$ \\
\hline $\mathrm{F}_{3}$ & $15,4 \pm 0,18^{b}$ & $16,8 \pm 0,04^{a}$ & $0,48 \pm 0,032^{b}$ & $110,7 \pm 3,7^{\mathrm{c}}$ & $2,66 \pm 0,37^{d}$ \\
\hline $\mathrm{F} 5_{7}$ & $17,5 \pm 0,18^{\mathrm{d}}$ & $21,2 \pm 0,49^{\mathrm{a}, \mathrm{b}, \mathrm{c}}$ & $0,32 \pm 0,018^{a}$ & $97,1 \pm 8,8^{b}$ & $2,70 \pm 0,32^{d}$ \\
\hline
\end{tabular}

Média \pm desvio padrão ( 3 repetições). Nota: letras diferentes, na mesma coluna, representam diferenças significativas $(\mathrm{p}>0,05)$ entre as médias obtidas através do Teste de Tukey. 
Tabela 3. Permeabilidade ao vapor de água (PVA) de Filmes F1 e F5.

\begin{tabular}{lcc}
\hline Filme & $\begin{array}{c}\text { Espessura } \\
(\mathbf{m m})\end{array}$ & $\begin{array}{c}\text { PVA } \\
[\text { g.mm/(m².dia.kPa })]\end{array}$ \\
\hline $\mathrm{F}_{3}$ & $0,057 \pm 0,004^{\mathrm{a}}$ & $4,28 \pm 0,43^{\mathrm{a}}$ \\
$\mathrm{F}_{7}$ & $0,061 \pm 0,004^{\mathrm{a}}$ & $4,79 \pm 0,28^{\mathrm{a}, \mathrm{b}}$ \\
$\mathrm{F}_{3}$ & $0,064 \pm 0,005^{\mathrm{a}}$ & $4,15 \pm 0,16^{\mathrm{a}}$ \\
$\mathrm{F}_{7}$ & $0,062 \pm 0,003^{\mathrm{a}}$ & $4,63 \pm 0,14^{\mathrm{a}}$ \\
$\mathrm{F}_{3}$ & $0,063 \pm 0,004^{\mathrm{a}}$ & $4,42 \pm 0,16^{\mathrm{a}}$ \\
$\mathrm{F5}_{7}$ & $0,062 \pm 0,002^{\mathrm{a}}$ & $5,34 \pm 0,23^{\mathrm{b}}$ \\
\hline
\end{tabular}

Média \pm desvio padrão (3 repetições). Nota: letras diferentes, na mesma coluna, representam diferenças significativas $(p>0,05)$ entre as médias obtidas através do teste de Tukey.

tenham utilizado filmes de maior espessura e uma metodologia de reticulação diferente, a tendência foi a mesma encontrada neste trabalho.

$\mathrm{Na}$ Tabela 3, são apresentados os valores da espessura e do coeficiente de permeabilidade ao vapor de água obtidos para filmes representativos. Os resultados indicam que a reticulação com $7 \%$ de $\mathrm{CaCl}_{2} \cdot 2 \mathrm{H}_{2} \mathrm{O}$ no $2^{\circ}$ estágio aumenta ligeiramente a permeabilidade ao vapor de água. De acordo com a classificação estabelecida por Krochta e De Mulder-Johnston ${ }^{[30]}$, o coeficiente de permeabilidade ao vapor de água obtido pode ser considerado como moderado, comparado a filmes sintéticos. Os valores de $P V A$ encontrados são da mesma ordem de grandeza dos encontrados em filmes obtidos com outros biopolímeros. Zactiti e Kieckbusch ${ }^{[21]}$ encontraram valores ligeiramente maiores de permeabilidade. Carvalho e Grosso ${ }^{[31]}$ constataram que filmes de gelatina $(0,10 \mathrm{~g}$ de gelatina/mL de solução) apresentam permeabilidade ao vapor de água de aproximadamente $4,9 \pm 0,17 \mathrm{~g} . \mathrm{mm} / \mathrm{m}^{2}$.dia.kPa, utilizando umidade relativa igual a $50 \%$, em filmes com espessura de $0,08 \pm 0,004 \mathrm{~mm}$. Filmes à base de pectina $(0,04 \mathrm{~g}$ de pectina/mL de solução) com espessura igual a $0,07 \mathrm{~mm}$ na temperatura de $25^{\circ} \mathrm{C}$ e umidade relativa a $75 \%$ apresentaram uma $P V A$ igual a $6,80 \pm 0,12$ g.mm $/ \mathrm{m}^{2} \cdot \operatorname{dia} \cdot \mathrm{kPa}^{[32]}$.

\section{Cinética de liberação do benzoato de cálcio}

A curva de liberação de benzoato de cálcio de filmes reticulados com $3 \%$ de solução de cloreto de cálcio é mostrada na Figura 1. A curva de liberação segue uma cinética de $1^{\text {a }}$ ordem, apresentando uma taxa inicial extremamente alta. Analisando os resultados obtidos, verifica-se que $95 \%$ da massa é transferida nos primeiros 4 minutos, isto é, ainda durante o período de intumescimento, podendo concluir, portanto, que o filme produzido tem baixo controle de liberação. As condições do ensaio, entretanto, foram extremamente adversas. Ao atuar como embalagem de alimentos, a difusão ocorre apenas por uma das faces (a liberação é 4 vezes mais lenta), pode haver uma resistência de contato entre o filme e o alimento e a concentração de benzoato na superfície não será zero, mas dependerá da capacidade de absorção por parte do alimento, e o filme, a não ser que esteja em contato com um alimento muito úmido, não estará intumescido pelo contato com água pura.

Todos os dados experimentais foram ajustados a Equação 3 com 21 termos no somatório e a difusividade efetiva média global foi calculada usando a espessura final do filme (equilíbrio). Esses valores, bem como os coeficientes de correlação encontrados para cada situação estudada estão apresentados na Tabela 4. As linhas traçadas na Figura 1 correspondem à solução da $2^{\mathrm{a}}$ Lei de Fick.

Os resultados indicam um aumento da difusividade efetiva com o aumento da concentração de benzoato no filme. Zactiti e Kieckbusch $^{[21]}$ encontraram comportamento semelhante estudando

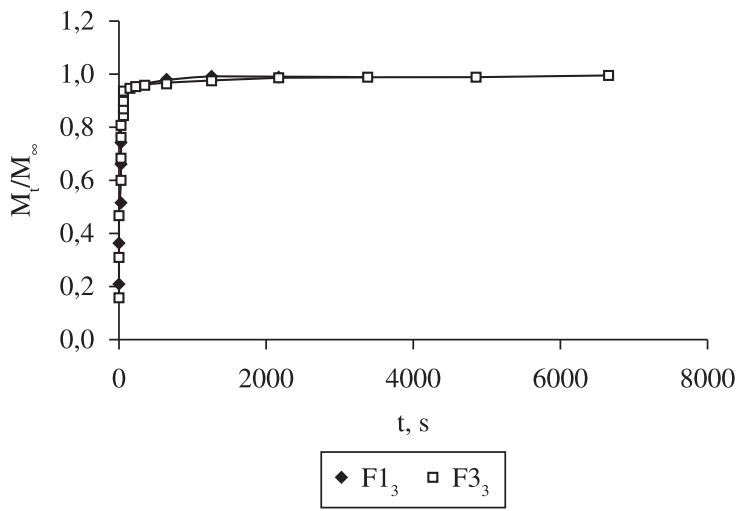

Figura 1. Fração de benzoato liberada de filmes reticulados com solução de $3 \% \mathrm{CaCl}_{2} 2 \mathrm{H}_{2} \mathrm{O}: \diamond \mathrm{F}_{3} \mathrm{e} \square \mathrm{F} 3$.

Tabela 4. Coeficiente de difusão efetivo do benzoato de cálcio $\left(D_{\text {ef }}\right.$ calculado pela Equação 3 ) em filmes com diferentes concentrações iniciais de benzoato de cálcio e reticulados com 3 ou $7 \% \mathrm{CaCl}_{2} \cdot 2 \mathrm{H}_{2} \mathrm{O}$.

\begin{tabular}{|c|c|c|c|}
\hline Filme & $\delta_{\mathrm{f}}(\mathbf{m m})$ & $\mathbf{R}^{2}$ & $D_{e f} \times 10^{7}\left(\mathrm{~cm}^{2} / \mathrm{s}\right)$ \\
\hline $\mathrm{F}_{3}$ & 0.094 & 0.9991 & 4.23 \\
\hline $\mathrm{F} 1_{7}$ & 0.074 & 0.9995 & 3.21 \\
\hline $\mathrm{F}_{3}$ & 0.117 & 0.9996 & 5.93 \\
\hline $\mathrm{F} 3_{7}$ & 0.108 & 0.9996 & 5.01 \\
\hline
\end{tabular}

a difusão de sorbato de potássio em filmes de alginato. A ordem de magnitude das difusividades encontradas está de acordo com os valores relatados na literatura para filmes de proteínas ${ }^{[20]}$. As difusividades de benzoato de cálcio em filmes de alginato são uma ordem de grandeza maior do que as difusividades encontradas em filmes de polietileno por Han e Floros ${ }^{[33]}$.

Os expoentes de difusão para o modelo da Lei da Potência (Equação 5) foram obtidos plotando $\ln \left(\mathrm{M}_{\mathrm{t}} / \mathrm{M}_{\infty}\right)$ contra $\ln (\mathrm{t})$. A linearização das duas situações estudadas com filmes reticulados com $3 \% \mathrm{CaCl}_{2}$ podem ser vistas na Figura 2, e os coeficientes de correlação obtidos foram excelentes $\left(\mathrm{R}^{2}>0,999\right)$. O expoente difusional (n) foi calculado a partir da inclinação destas linhas, enquanto que a constante de difusão (k) foi obtida a partir da interceptação. Como mostrado na Tabela 5, os valores do expoente difusional variaram entre $0.5 \mathrm{e}$ 1.0, sugerindo que o processo de transporte tinha características não-Fickianas (anômalo) e uma taxa de difusão do solvente da mesma ordem de grandeza que o relaxamento do polímero. O desvio do comportamento Fickiano indica que o fenômeno de relaxamento da estrutura do polímero é notório e pode afetar a liberação de benzoato de cálcio durante o período inicial do processo de difusão. Devido ao visível grau de intumescimento, consequiência da natureza hidrofílica da matriz investigada, as macromoléculas do polímero foram rearranjadas em uma nova conformação. Este pode ser o processo principal responsável pelo desvio do comportamento ideal da difusão Fickiana ${ }^{[34]}$.

O comportamento de difusão durante os experimentos de liberação até o equilíbrio pode ser comparado com a difusão de benzoato de cálcio durante o intenso período de intumescimento. Isto pode ser feito comparando os parâmetros de solução de tempos curtos com as difusividades efetivas globais já calculados e apresentados na Tabela 4. Os dados experimentais obtidos durante os primeiros 30 segundos de contato foram ajustados ao modelo de difusão para tempos curtos, através de um gráfico linear de $M_{t} / M_{\infty}$ vs. $\sqrt{t}$. A linearização das duas situações estudadas pode ser vista na Figura 3 e os parâmetros de ajuste são apresentados na Tabela 6 . 


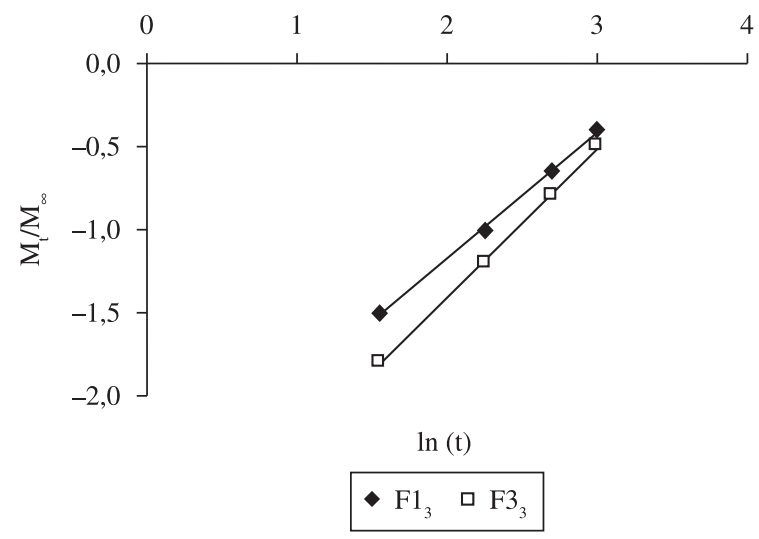

Figura 2. Modelo da Lei da Potência aplicado para a fração de benzoato liberada em filmes reticulados com solução de $3 \% \mathrm{CaCl}_{2} \cdot 2 \mathrm{H}_{2} \mathrm{O}: \diamond \mathrm{F}_{3} \mathrm{e} \square \mathrm{F} 3_{3}$.

Tabela 5. Expoente difusional (n), constante difusional (k) e coeficiente de correlação $\left(\mathrm{R}^{2}\right)$ de liberação de benzoato, modelado pela equação da Lei da Potência. Filmes reticulados com 3 ou $7 \% \mathrm{CaCl}_{2} \cdot 2 \mathrm{H}_{2} \mathrm{O}$.

\begin{tabular}{cccc}
\hline Filme & $\mathbf{n}$ & $\mathbf{k} \times \mathbf{1 0}^{\mathbf{2}}$ (segundos) & $\mathbf{R}^{\mathbf{2}}$ \\
\hline $\mathrm{F} 1_{3}$ & 0.8148 & 2.8659 & 0.9981 \\
$\mathrm{~F} 1_{7}$ & 0.9042 & 3.0361 & 0.9975 \\
$\mathrm{~F} 3_{3}$ & 0.9504 & 3.3459 & 0.9998 \\
$\mathrm{~F} 3_{7}$ & 0.8900 & 3.1414 & 0.0072 \\
\hline
\end{tabular}

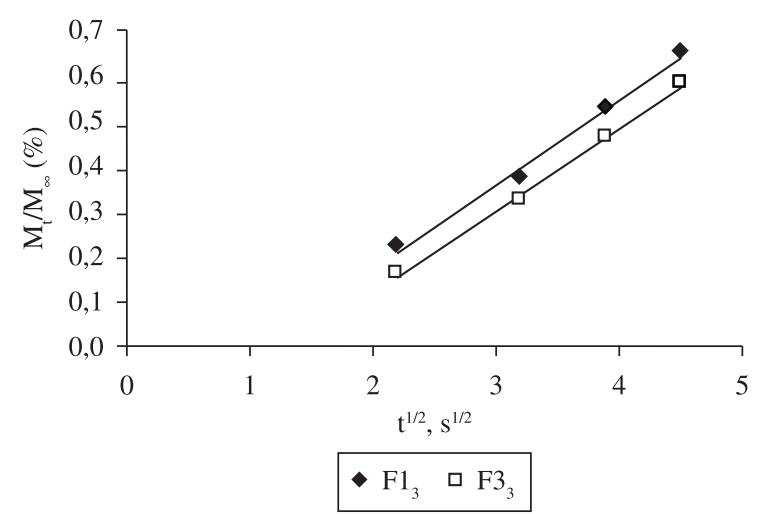

Figura 3. Ajuste dos dados de liberação do benzoato a solução de tempos curtos (reta contínua) para filmes reticulados com solução de $3 \%$ $\mathrm{CaCl}_{2} 2 \mathrm{H}_{2} \mathrm{O}: \diamond \mathrm{F}_{3} \mathrm{e} \square \mathrm{F}_{3}$

Tabela 6. Coeficiente angular (a), coeficiente linear (b) e coeficiente de correlação $\left(\mathrm{R}^{2}\right)$ obtidos a partir de um gráfico linear de $\mathrm{M}_{t} / \mathrm{M}_{\infty} \times \sqrt{t}$. Filmes reticulados com 3 ou $7 \% \mathrm{CaCl}_{2} \cdot 2 \mathrm{H}_{2} \mathrm{O}$.

\begin{tabular}{cccc}
\hline Filme & a & b & $\mathbf{R}^{2}$ \\
\hline $\mathrm{F}_{3}$ & 0.2008 & -0.2508 & 0.9893 \\
$\mathrm{~F}_{7}$ & 0.2090 & -0.2639 & 0.9999 \\
$\mathrm{~F}_{3}$ & 0.1971 & -0.2908 & 0.9938 \\
$\mathrm{~F}_{7}$ & 0.1916 & -0.2539 & 0.9996 \\
\hline
\end{tabular}

De acordo com a Equação 4, a interceptação da abscissa deve ser zero. Os valores encontrados correspondem a uma diferença inferior a 2 segundos, o que é aceitável e pode corresponder a uma extração contínuada de benzoato durante a transferência sequencial das amostras de um copo para outro, já que uma pequena película de umidade permanecia na superfície do filme.

A difusividade do benzoato no filme foi calculada usando o coeficiente linear e a espessura média dos filmes. Os resultados
Tabela 7. Difusividade efetiva $\left(\mathrm{D}_{\mathrm{ef}}\right)$ de benzoato, calculado pela solução de tempos curtos. Espessura inicial $\left(\delta_{\mathrm{i}}\right)$, espessura final $\left(\delta_{\mathrm{f}}\right)$ e espessura média $\left(\delta_{\mathrm{m}}\right)$. Filmes reticulados com 3 ou $7 \% \mathrm{CaCl}_{2} \cdot 2 \mathrm{H}_{2} \mathrm{O}$.

\begin{tabular}{ccccc}
\hline Filme & $\delta_{\mathbf{i}}(\mathbf{m m})$ & $\delta_{\mathbf{f}}(\mathbf{m m})$ & $\delta_{\mathbf{m}}(\mathbf{m m})$ & $\mathbf{D}_{\mathbf{e f}} \times \mathbf{1 0}^{\mathbf{7}}\left(\mathbf{c m}^{2} / \mathbf{s}\right)$ \\
\hline $\mathrm{F}_{3}$ & 0.059 & 0.094 & 0.077 & 4.69 \\
$\mathrm{~F}_{7}$ & 0.053 & 0.074 & 0.064 & 3.51 \\
$\mathrm{~F}_{3}$ & 0.064 & 0.117 & 0.091 & 6.32 \\
$\mathrm{~F}_{7}$ & 0.064 & 0.108 & 0.086 & 5.33 \\
\hline
\end{tabular}

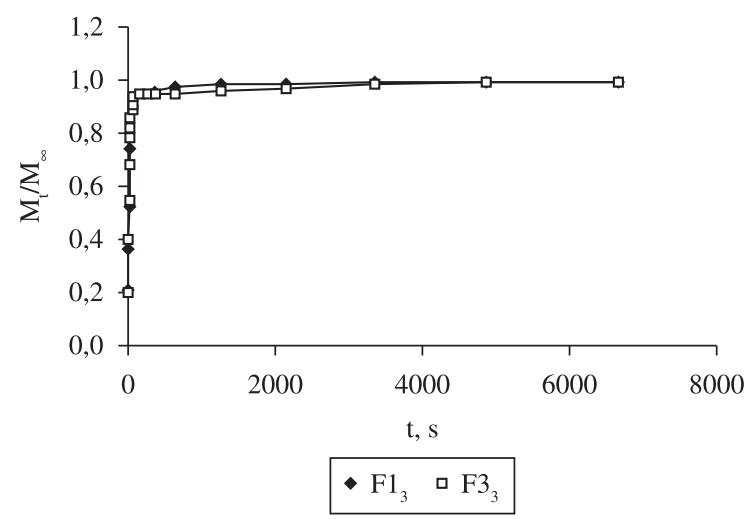

Figura 4. Fração de benzoato liberada do filme F1 reticulado com solução de 3 ou $7 \% \mathrm{CaCl}_{2} \cdot \mathrm{H}_{2} \mathrm{O}: \diamond \mathrm{F}_{3} \mathrm{e} \square \mathrm{F}_{7}$.

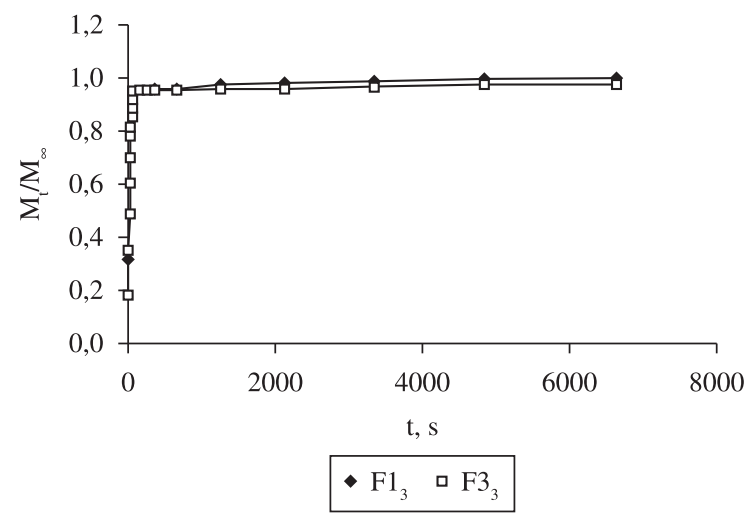

Figura 5. Fração de benzoato liberada do filme F3 reticulado com solução de 3 ou $7 \%$ de $\mathrm{CaCl}_{2} \cdot \mathrm{H}_{2} \mathrm{O}: \diamond \mathrm{F}_{3} \mathrm{e} \square \mathrm{F}_{7}$.

estão apresentados na Tabela 7. Uma comparação entre os valores da Tabela 7 (difusividade inicial) e na Tabela 4 (difusividade média global) mostra que eles são suficientemente próximos, permitindo concluir que o processo de absorção de água tem uma influência perceptível, mas pequena em difusividade. É importante observar, no entanto, que esses valores são fortemente influenciados pela precisão da determinação de espessura e um modelo mais realista deve incluir a variação do tempo em $\delta$ na integração da $2^{\text {a }}$ Lei de Fick.

Resultados semelhantes foram obtidos por Ozdemir e Floros ${ }^{[20]}$, que investigaram a difusão de sorbato de potássio em filmes de proteínas. Eles encontraram que o comportamento não-Fickiano foi o mecanismo predominante no início do processo de liberação do soluto e desenvolveram um modelo matemático, derivado da $2^{\mathrm{a}}$ Lei de Fick, combinando as duas difusões em contracorrente, a entrada da água e a saída de benzoato. Bodea e Leucuta ${ }^{[35]}$ estudaram um processo semelhante de liberação de filmes poliméricos ativos, sintetizados com uma mistura de dois polímeros hidrofílicos: hidroxipropilmetilcelulose e carboximetilcelulose de sódio. Os resultados confirmaram difusão 
Fickiana, mas uma fração importante da substância ativa foi liberada devido ao relaxamento do polímero (difusão anômala).

Quando os filmes contendo benzoato de cálcio foram reticulados em contato com solução de cloreto de cálcio, com concentração mais elevada (7\%), uma cinética de liberação de primeira ordem similar a da Figura 1 foi observada e os resultados estão apresentados nas Figuras 4 e 5.

Excelentes ajustes do modelo da Lei da Potência foram novamente obtidos, e o expoente difusional e a contante difusional calculados estão apresentados na Tabela 5. A diminuição do expoente de difusão sugere que, com um aumento no grau de reticulação o mecanismo de transporte tende a aproximar das condições Fickianas.

Os valores obtidos para a difusividade efetiva média global e para a difusividade efetiva calculada para o período inicial de liberação (Tabela 7) tendem a diminuir com o aumento da concentração de $\mathrm{Ca}^{2+}$, indicando que a mobilidade do benzoato foi afetada pelo aumento do grau de reticulação.

\section{Conclusões}

O transporte de benzoato de cálcio em filmes de alginato segue comportamento Fickiano e não-Fickiano. A correlação obtida com o ajuste ao modelo de tempos curtos indica que uma aproximação Fickiana é aceitável. Os valores de difusividade efetiva global obtidos usando o modelo derivado da $2^{\mathrm{a}}$ Lei de Fick são próximos aos valores obtidos para a liberação inicial, inferindo que o processo de intumescimento tenha uma influência limitada sobre o processo de difusão do benzoato, provavelmente devido aos efeitos compensadores: a resistência devido ao escoamento de água em contracorrente foi minimizada pela relaxação da estrutura polimérica. Os valores de difusividade encontrados são uma ordem de grandeza menor que a difusividade em alimentos com umidade intermediária, indicando um uso potencial para filmes de alginato como sistemas de liberação de substâncias ativas.

\section{Agradecimentos}

Ao Conselho Nacional de Desenvolvimento Científico e Tecnológico (CNPq) pela concessão da bolsa de mestrado e a FAPESP pelo auxílio à pesquisa (Proc. 2005/55499-3).

\section{Referências Bibliográficas}

1. Scott, G. - Polym. Degrad. Stab., 68, p.1 (2000).

2. Buonocore, G. G.; Conte, A.; Corbo, M. R.; Sinigaglia, M. \& Del Nobile, M. A. - Innov. Food Sci. Emerg. Technol., 6, p.459 (2005).

3. Cherian, G.; Gennadios, A.; Weller, C. \& Chinachoti, P. Cereal Chemist., 72, p.1 (1995)

4. Han, J. H. - Food Technol., 54, p.56 (2000).

5. Quattara, B.; Simard, R.; Piette, G.; Begin, A. \& Holley, R. - J. Food Sci., 65, p.768 (2000).

6. Coma, V.; Sebti, I.; Pichavant, F.; Pardon, P. \& Deschamps, A. - J. Food Protec., 64, p.470 (2001).

7. King, A. \& Brown, H. - Food Hydrocoll., 2, p.115 (1983).

8. Moe, S. T.; Draget, K. I.; Skjak-Braek, G. \& Smidsrød, O. - "Alginates", in: Food polysaccharides and their applications, A. M. Stephen (Ed.), Marcel Dekker, New York (1995).
9. Ikeda, A., Takemura, A. \& Ono, H. - Carbohyd. Polym., 42, p.421 (2000).

10. Grant, G. T.; Morris, E. R.; Rees, D. A.; Smith, P. J. C. \& Thom, D. Febs Lett., 32, p.195 (1973).

11. King, A. \& Brown H. - Food Hydrocoll., 2, p.115 (1983).

12. Mancini, M.; Moresi, M. \& Rancini, R. - J. Food Eng., 39, p.369 (1999).

13. Turbiani, F. R. B. - "Desenvolvimento e caracterização de filmes ativos de alginato de sódio reticulados com benzoato de cálcio", Dissertação em Engenharia Química, Faculdade de Engenharia Química, Universidade Estadual de Campinas, Campinas (2007).

14. Rhim, J. W.; Gennadios, A.; Weller, C. L. \& Hanna, M. A. - Indust. Crops Prod., 15, p.199 (2002).

15. Gontard, N.; Guilbert, S. \& Cuq, J. L. - J. Food Sci., 58, p.206 (1993).

16. Bigi, A.; Panzavolta, S. \& Rubini, K. - Biomaterials, 25, p.5675 (2004).

17. ASTM International - "D 82: Tensile properties of thin plastic sheeting", Philadelpia (1995).

18. ASTM International - "E 96: Standard test methods of water vapor transmission of materials", Philadelphia (1995).

19. Crank, J. - "The mathematics of diffusion", $2^{\text {nd }}$ ed., Clarendon Press, Oxford (1975)

20. Ozdemir, M. \& Floros, J. D. Analysis and modeling of potassium sorbate diffusion through edible whey protein films. J. Food Eng., 47, p.149-155 (2001).

21. Zactiti, E. M. \& Kieckbusch, T. G. - J. Food Eng., 77, p.462 (2006).

22. Pavlath, A. E.; Grossett, C.; Camirand, W. \& Robertson, G. H. - J. Food Sci., 64, p.61 (1999).

23. Rajsharad, C. \& Kamble, S. - "Aqueous film coating containing sodium alginate and preparation thereof", Patent - IPC8 Class: AA61K4736FI, USPC Class: 514779-12/(2008).

24. Kim, K. W.; Ko, C. J. \& Park, H. J. - J. Food Sci., 67, p.218 (2002).

25. Rhim, J. W. - Lebensm. Wiss. Technol., 37, p.323 (2004).

26. Al-Musa, S.; Abu Fara, D. \& Badwan, A. A. - J. Control. Release, 57, p.223 (1999).

27. Zactiti, E. M. \& Kieckbusch, T. G. - Packag. Technol. Sci., 22, p.349 (2009).

28. Smidsrød, O. - Faraday Discuss. Chem. Soc., 57, p.263 (1974).

29. Resmuñan-López, C. \& Bodmeier, R. - J. Controll. Release, 44, p.215 (1997).

30. Krochta, J. M. \& Mulder-Johnston, C. D. - Food Technol., 51, p.61 (1997).

31. Carvalho, R. A. \& Grosso, C. R. F. - J. Chem. Eng., 23, p.45 (2006).

32. Batista, J. A.; Tanada-Palmu, P. S. \& Grosso, C. R. F. - Cien. Tecnol. Aliment., 25, p.781 (2005).

33. Han, J. H. \& Floros, J. D. - J. Food Sci., 63, p.435 (1998).

34. Flores, S.; Conte, A.; Campos, C.; Gerschenson, L. \& Del Nobile, M. - J. Food Eng., 81, p.580 (2007).

35. Bodea, A. \& Leucuta, S. E. - Inter. J. Pharm., 153, p.247 (1997).t

Enviado: 02/10/09

Reenviado: $18 / 08 / 10$

Aceito: $25 / 10 / 10$

DOI: $10.1590 / \mathrm{S} 0104-14282011005000034$ 